\title{
Multimodal Control of Liquid Crystalline Mesophases from Surfactants with Photoswitchable Tails
}

Received 00th January 20xx, Accepted 00th January 20xx

DOI: $10.1039 / x 0 x x 00000 x$

\author{
Judith E. Houston, ${ }^{\mathrm{a}, \mathrm{b}, \ddagger}$ Elaine A. Kelly, ${ }^{\mathrm{b}}$ Margarita Kruteva, ${ }^{\mathrm{c}}$ Kiriaki Chrissopoulou, ${ }^{\mathrm{d}}$ Nathan Cowieson ${ }^{\mathrm{e}}$ \\ and Rachel C. Evans ${ }^{\mathrm{f}}$
}

\begin{abstract}
Non-invasive manipulation of the hierarchical structure of functional materials is a key challenge in the advancement of optoelectronics, energy conversion and storage devices and drug delivery systems. Lyotropic liquids crystals, with their longrange order and a rich array of structures have long been exploited as soft templates to prepare porous materials. The potential to modify the template structure using an external stimulus such as light could lead to new architectures. Here, using a combination of small-angle X-ray scattering and polarised optical microscopy, we decipher the various structure/selfassembly relationships of neutral surfactants bearing photoswitchable tails, which self-organise into a rich variety of lyotropic liquid crystalline mesophases. Facile, multimodal control of the nanoscale morphology of these single-component systems is achieved through: i) molecular structure, via careful selection of the alkyl tail/ethylene oxide headgroup lengths; ii) concentration; iii) temperature; and iv) photoisomerisation. The nanoscale architectures range from the weakly concentrated hyperswollen lamellar phases, the more common lyotropic lamellar and hexagonal phases, to pure thermotropic liquid crystals; all of which are accessible at room temperature. Photoisomerisation with UV light leads to the reversible destruction of the liquid crystalline phase, which can be spatially controlled through the use of a mask. This extensive study demonstrates the versatility of neutral photosurfactants and paves the way for them to be investigated for new applications, such as photoresponsive templates or drug delivery systems.
\end{abstract}

\section{Introduction}

Molecular self-assembly is an elegant approach for the fabrication of nanostructured materials with well-defined dimensions and morphology from the bottom up. Lyotropic liquid crystalline (LLC) phases, derived from the concentration-dependant self-assembly of surfactants, have been frequently exploited for this purpose, both in their native form, for example as vessels for drug delivery ${ }^{1,2}$ and as optical modulators, ${ }^{3}$ or as soft templates for the construction of porous materials. ${ }^{4-8}$ LLC phases show long-range periodicity and exhibit a rich polymorphism of structures, whose mesoscale organisation depends critically on the molecular structure of the surfactant and the local packing. ${ }^{9}$ As such, access to LLC mesophases with tailored dimensions requires the informed design of new amphiphiles, whose structure-packing relationships are wellunderstood.

Particular potential lies in the design of LLC assemblies that incorporate added functionality, either as an intrinsic property, e.g. electrical conductivity, ${ }^{10}$ or as a response to an external trigger, such

\footnotetext{
a. Jülich Centre for Neutron Science (JCNS-4), Forschungszentrum, Jülich GmbH, Lichtenbergstr. 1, 85748 Garching, Germany

b. School of Chemistry and CRANN, University of Dublin, Trinity College, College Green, Dublin 2, Ireland.

Jülich Centre for Neutron Science (JCNS-1) and the Institute for Complex Systems (ICS).

d. Institute of Electronic Structure and Laser, Foundation for Research and Technology, Hellas, P.O. Box 1527, 711 10, Heraklion, Crete, Greece.

. Diamond Light Source, Harwell Science and Innovation Campus, Didcot, Oxfordshire, OX11 ODE, U.K.

f. Department of Materials Science \& Metallurgy, University of Cambridge, 27 Charles Babbage Road, Cambridge, CB3 OFS, U.K. Email: rce26@cam.ac.uk

† Electronic Supplementary Information (ESI) available: Instrumentation, synthesis and characterization of photosurfactants, critical micelle concentrations, packing parameters, small-angle X-ray scattering and polarised optical microscopy, hyperswollen lamellar phases, photoisomerisation studies, binary temperatureconcentration phase diagrams. See DOI: 10.1039/x0xx00000x

‡ Current address: European Spallation Source ERIC, Box 176, SE-221 00 Lund, Sweden.
}

as light, ${ }^{11}$ magnetic fields ${ }^{12}$ or temperature. ${ }^{13}$ Light is commonly used as a stimulus as it can be applied with spatial and temporal control. Photoresponsive LLCs have been prepared through the addition of additives, such as gold nanoparticles ${ }^{14}$ or photochromic molecules such as spiropyran. ${ }^{15}$ In contrast, light-responsive LLC mesophases constructed from surfactants which bear chromophoric units as part of their molecular structure are far less common. ${ }^{16-19}$ This is surprising, as single component systems arguably hold the greatest potential to be developed for new bio-friendly and environmentallyfriendly multifunctional materials.

Azobenzene is commonly used as a prototypical molecular switch as it undergoes a trans-cis photoisomerisation under UV irradiation, ${ }^{20,} 21$ which can be reversed over many cycles with either blue light or heat.22-24 Upon incorporation into a surfactant, photoisomerisation leads to a substantial change in the geometry, hydrophilicity and packing of azobenzene photosurfactants (AzoPS) at both the molecular and micellar level. ${ }^{25-27}$ However, while conventional surfactants self-assemble into a variety of LLC polymorphs at concentrations significantly above the critical micelle concentration (cmc), reports of LLC phase formation in pure AzoPS systems are still rare. ${ }^{16-19}$ Hughes et al. reported LLC phase formation for charge-neutral AzoPS consisting of a hydrophilic oligooxyethylene unit, a hydrophobic alkyl chain and the azobenzene unit $\left(\mathrm{C}_{4+x} \mathrm{AzoC}_{y} \mathrm{E}_{7}\right.$, where $x=0, y=5$ or $x=5, y=0) .{ }^{16}$ Using differential scanning calorimetry, polarised optical microscopy (POM), small-angle X-ray scattering (SAXS) and rheology studies, they showed that the LLC phase formed could be controlled by varying the position of the azobenzene moiety within the hydrophobic tail. Furthermore, photoisomerisation of the $\mathrm{C}_{4} \mathrm{AzO}_{5} \mathrm{C}_{7}$ homologue significantly changed the LLC structure and its bulk properties. While these studies reveal an exciting opportunity to produce dynamic, nanoorganized arrays in solution using AzoPS, the fundamental molecular structure-packing relationships have not been explored or understood in detail. 


\section{Trans-AzoPS}

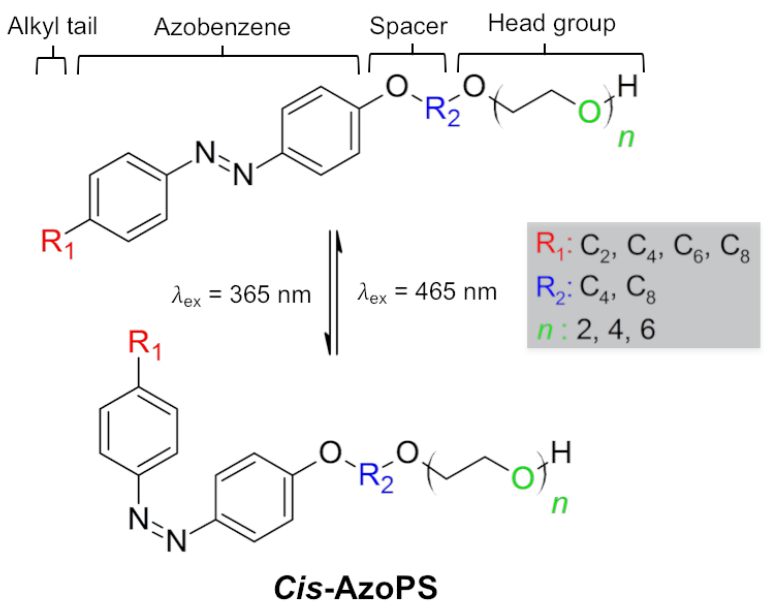

Figure 1. Molecular structure of the AzoPS investigated. $R_{1}$ and $R_{2}$ correspond to the alkyl tail and spacer, respectively, and $n$ indicates the number of oxyethylene units in the hydrophilic head group.

Herein, we demonstrate the combined use of molecular design with multimodal control to access diverse AzoPS LLC phases. Photosurfactant structures were first designed using the simple packing parameter model, ${ }^{28} P=V / a_{\mathrm{e}} l_{\mathrm{c}}$, where $V$ and $I_{\mathrm{c}}$ are the volume and length of the hydrophobic tail, respectively, and $a_{\mathrm{e}}$ is the area occupied by the headgroup at the hydrophilic/hydrophobic interface. Molecular structures with different head-to-tail volumes were targeted, as they were expected to self-assemble into aggregates with different degrees of curvature. As shown in Figure 1, each AzoPS has four main components: (i) ethylene oxide (E) as the hydrophilic head-group $\left(E_{2}, E_{4}\right.$ or $\left.E_{6}\right)$ and a hydrophobic tail consisting of (ii) an alkyl spacer $\left(\mathrm{C}_{4} \mathrm{H}_{8}\right.$ or $\left.\mathrm{C}_{8} \mathrm{H}_{16}\right)$ between the hydrophilic head and (iii) the photoresponsive azobenzene unit; (iv) a hydrophobic alkyl tail group $\left(\mathrm{C}_{2} \mathrm{H}_{5}, \mathrm{C}_{4} \mathrm{H}_{9}, \mathrm{C}_{6} \mathrm{H}_{13}\right.$ or $\left.\mathrm{C}_{8} \mathrm{H}_{17}\right)$. Full details of the synthesis and characterisation of each AzoPS and all intermediates can be found in the Electronic Supplementary Information (ESI). We observe that while the packing parameter model neglects the shape of the hydrophobic tail, 29 it provides a reasonable prediction of the aggregate shape and expected mesophase for the trans-isomers of each structure. Harnessing concentration, temperature, structural variations and light, we show that it is possible to access a variety of different LLC phases, as well as thermotropic liquid crystals. Moreover, irradiation with UV light results in destruction of the LLC phase due to transformation to the cis-isomer at the molecular level. This suggests that the simple packing parameter cannot take into account the dramatic volume change associated with the trans-cis photoisomerisation of the azobenzene (Figure 1). ${ }^{30}$ Notably, we show that following photodeformation, the original LLC phase can easily be recovered through gentle heating or irradiation with blue light.

\section{Experimental section}

\section{Synthesis}

The synthesis of each AzoPS proceeded via the same general approach, achieved through modifications of previous literature procedures: ${ }^{31,32}(1)$ the preparation of a hydroxyl precursor, 4alkyl-4'-hydroxylazobenzene (via diazotisation and azocoupling); (2) modification of the above to a bromoprecursor, 4alkyl-4'-(2-bromo)alkyloxy azobenzene, via an $\mathrm{S}_{\mathrm{N}} 2$ reaction; (3) addition of the neutral ethylene glycol head groups $\left(E_{2}, E_{4}, E_{6}\right)$ to yield the final product. Comprehensive experimental details and characterisation data $\left({ }^{1} \mathrm{H} N \mathrm{NMR},{ }^{13} \mathrm{C}\right.$ NMR, Fourier-transform infrared spectroscopy, high-resolution mass spectrometry, melting point analysis) are available for each product and intermediate in the ESI.

\section{UV/Vis absorption spectroscopy}

UV/Vis absorption spectra were recorded at room temperature on a Shimadzu UV2401 PC UV-Vis scanning spectrometer with a scanning slit width of $0.5 \mathrm{~nm}$. The UV/Vis absorption experiments were carried out using an ILT 950 spectroradiometer over a range of $250-1050 \mathrm{~nm}$ with a resolution of $1.4 \mathrm{~nm}$ and $25 \mu \mathrm{m}$ slits. Optical data were recorded using SpectraLight III software. A DH-2000 deuterium tungsten halogen light source was used to produce light over 215-2000 $\mathrm{nm}$.

\section{Polarised optical microscopy}

Temperature-controlled POM measurements were either carried out at FORTH institute in Heraklion, Crete on a Zeiss Axio Scope.A.1 polarised optical microscope fitted with a $\mathrm{N}_{2}$ cooled heat-stage or at the Jülich Centre for Neutron Science at the Heinz Maier-Leibnitz Zentrum, Garching, Germany on a Leica DM 6000 Digital Microscope. A series of highly concentrated AzoPS samples (10-100 wt\%) were prepared and deposited between two glass cover slips. These samples were imaged using a polarised optical microscope, equipped with a liquid nitrogen-cooled heating stage, to probe the LLC phases formed over a range of temperatures. Each sample was slowly heated and cooled between $5-70^{\circ} \mathrm{C}$, using a heating rate of $10^{\circ} \mathrm{C} \mathrm{min}$ 1. Images were initially recorded in $5{ }^{\circ} \mathrm{C}$ intervals as the samples were heated. At temperatures where significant phase transitions were recorded repeat measurements were performed at $1{ }^{\circ} \mathrm{C}$ intervals, with a slower heating rate of $1{ }^{\circ} \mathrm{C} \mathrm{min}^{-1}$. Photoisomerisation of the AzoPS samples was achieved via 10 min of irradiation with a UV light-emitting diode $\left(\lambda_{\mathrm{ex}}=365 \mathrm{~nm}\right)$ for the trans-cis isomerisation and a blue lightemitting diode $\left(\lambda_{\mathrm{ex}}=465 \mathrm{~nm}\right)$ for the reverse cis-trans isomerisation. Control experiments were performed to ensure that no photoisomerisation would be triggered as a result of the halogen lamp in the optical microscope. A number of cisisomers were left on the light stage for $\sim 2 \mathrm{~h}$ and no change in texture of the image was observed in this time.

\section{Small-angle X-ray scattering (SAXS)}

Synchrotron SAXS measurements were performed on the BioSAXS beamline B21, Diamond Light Source, Harwell, United Kingdom. B21 operates in a fixed sample to detector distance $(4.014 \mathrm{~m})$ with $\mathrm{X}$-ray beam energy $E=12.4 \mathrm{keV}$ giving a $q$-range of 0.031-3.8 $\mathrm{nm}^{-1}$. The magnitude of the scattering vector $(q)$ is defined by:

$$
q=\frac{4 \pi}{\lambda} \sin \left(\frac{\theta}{2}\right)
$$


(a) $\mathrm{C}_{4} \mathrm{AzOOC}_{4} \mathrm{E}_{6}$

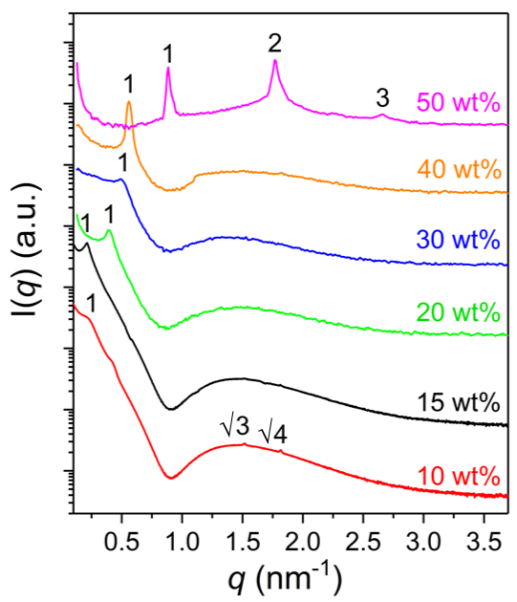

(b) $\mathrm{C}_{4} \mathrm{AzOOC}_{4} \mathrm{E}_{4}$

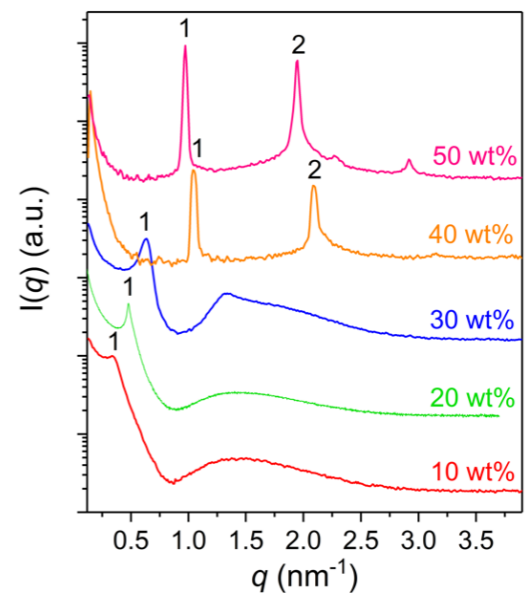

(c) $\mathrm{C}_{8} \mathrm{AzOOC}_{4} \mathrm{E}_{4}$

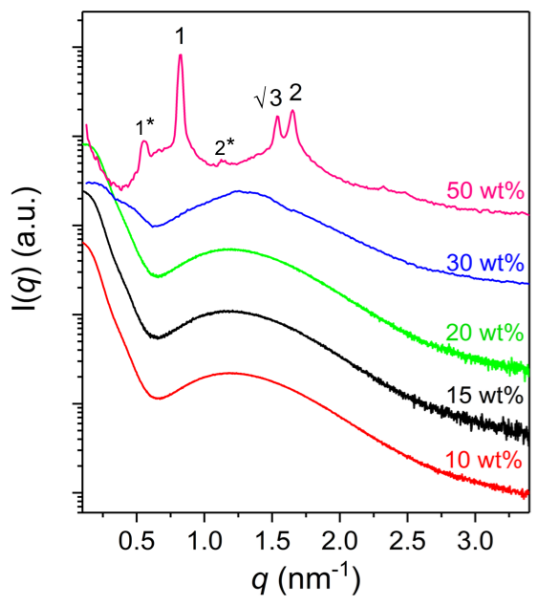

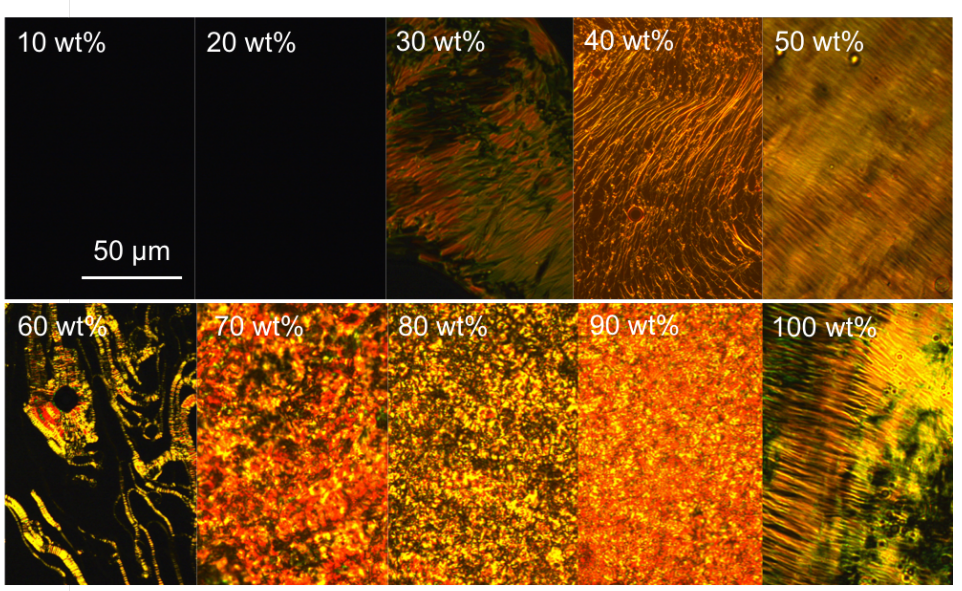
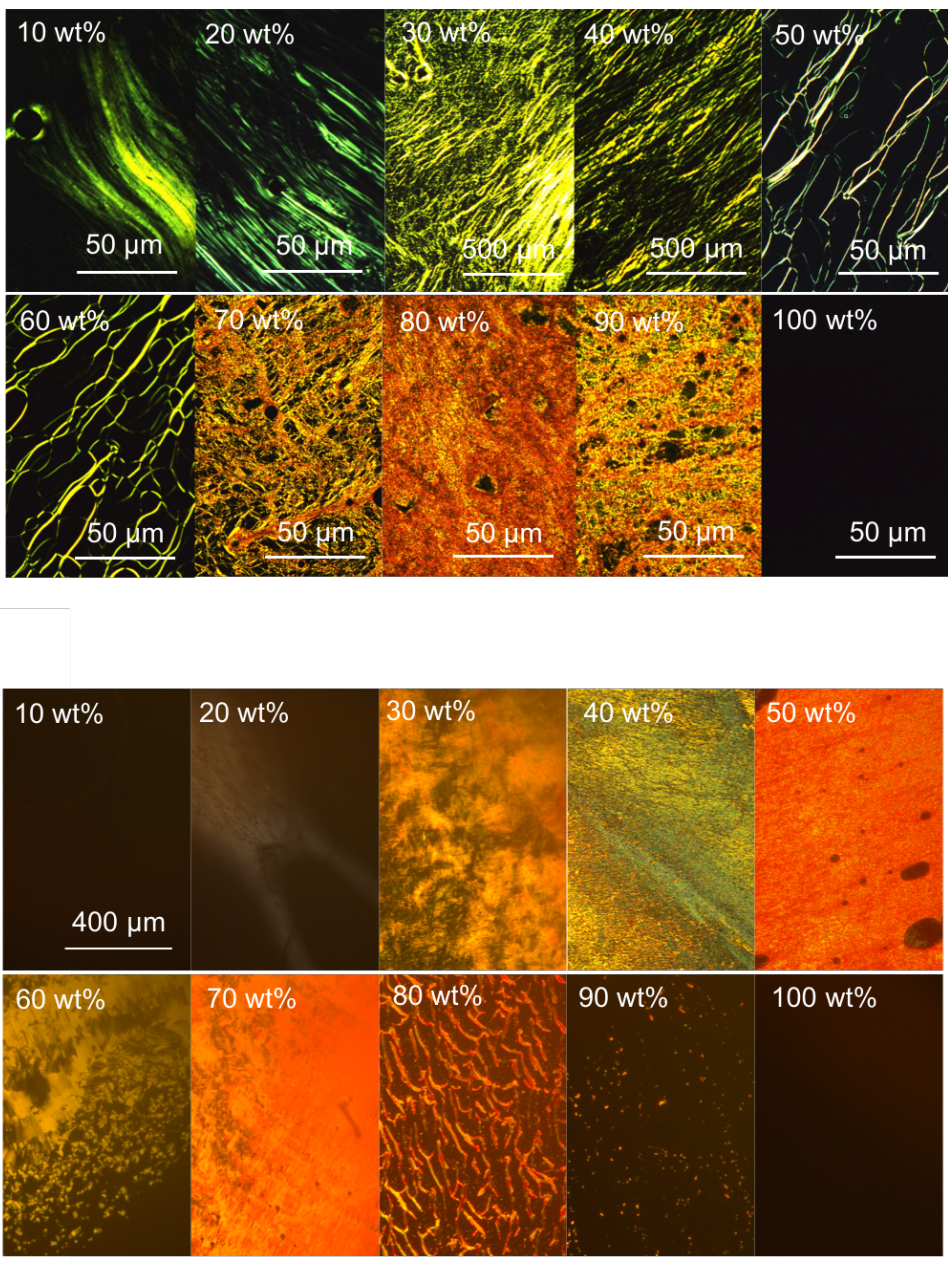

Figure 2. Lyotropic liquid crystalline phase formation by neutral AzoPS. SAXS patterns (left) and polarised optical micrographs (right) of (a) $\mathrm{C}_{4} \mathrm{AzoO} \mathrm{C}_{4} \mathrm{E}_{6}$, (b) $\mathrm{C}_{4} \mathrm{AzoOC}_{4} \mathrm{E}_{4}$ and (c) $\mathrm{C}_{8} \mathrm{AzoOC}_{4} \mathrm{E}_{4}$ as a function of concentration at $\mathrm{T}=20{ }^{\circ} \mathrm{C}$. Defined peaks in the SAXS profiles and brightness in the POM images indicate the formation of LLC phases. The SAXS curves have been offset for clarity. * indicates the presence of a second LLC phase.

where $\lambda$ is the wavelength of the $\mathrm{X}$-ray and $\vartheta$ is the scattering angle. Samples were loaded into PCR well plates in an automated sample changer. Samples $(30 \mu \mathrm{L})$ were then delivered into a temperature-controlled quartz capillary and exposed for $3 \mathrm{~s}$, collecting 60 frames at $20^{\circ} \mathrm{C}$. Data were collected using a Pilatus Dectris $2 \mathrm{M}$ detector. The background 
was manually subtracted using ScÅtter. Laboratory SAXS measurements were performed on the GALAXI beamline at the Jülich Centre for Neutron Science. ${ }^{33}$ GALAXI uses a Bruker AXS Metaljet $X$-ray source working with gallium $K_{\alpha} X$-rays $(\lambda=1.34$ $\AA$ ), and a sample-to-detector distance of $80 \mathrm{~cm}$, resulting in the $q$-range $0.1-7.0 \mathrm{~nm}^{-1}$. Data were collected using a Pilatus Dectris $1 \mathrm{M}$ detector. Samples $(\sim 50 \mu \mathrm{L})$ were inserted into borosilicate glass capillaries $(1.0 \mathrm{~mm})$, which were then sealed. The sampleto-detector distance was calibrated using the Bragg reflections from silver behenate.

\section{Chemical Structure Modelling Engine}

Three-dimensional (3D) models of chemical structures were generated on the free software $\mathrm{ACD} /$ ChemSketch $^{\mathrm{TM}} 3 \mathrm{D}$ viewer $^{34}$ and optimised using a 3D-optimisation algorithm which transforms planar 2D structures into realistic 3D structures. The algorithm is based on modified molecular mechanics, which consider bond stretching, angle bending, internal rotation and Van der Waals interactions. While this is not a full-scale molecular mechanics engine, the algorithm produces a reasonable estimation of the molecular conformation.

\section{Results and discussion}

The water-concentration phase behaviour of the AzoPS transisomers was first investigated in $10 \mathrm{wt} \%$ increments using a combination of POM and SAXS (Figure 2). The lowest concentration investigated (10 wt\%) is $\sim 1,000$ times greater than the cmcs of the AzoPS, which are summarised in Table S1 (ESI). Almost all trans-AzoPS exhibit the formation of at least one LLC phase at room temperature $\left(20^{\circ} \mathrm{C}\right)$. For example, at low concentrations (10-20 wt\%), the POM images of $\mathrm{C}_{4} \mathrm{AzoOC}_{4} \mathrm{E}_{6}$ are dark, indicating that the sample is isotropic (Figure 2a). However, as the concentration is increased to $60 \mathrm{wt} \%$, the POM images exhibit streaky or mosaic-like textures, attributed to lamellar mesophases $\left(L_{\alpha}\right) .{ }^{35}$ In general, for linear surfactants, the volume to length ratio of the tail $\left(V / I_{c}\right)$ will be almost constant and independent of tail length. On this basis, trans$\mathrm{C}_{4} \mathrm{AzoOC}_{4} \mathrm{E}_{6}$, which has the largest head group (and thus $a_{\mathrm{e}}$ ), is expected to have the smallest packing parameter $(P \sim 0.35$, see ESI for more details) suggesting it should form assemblies with high curvature (spheres/cylinders). However, due to the presence of the bulky azobenzene moiety within the tail, this AzoPS may be better represented as a truncated cone, which would indicate assemblies with lower curvature such as the lamellar sheets observed here. The speckled or fan-like textures of the 70-90 wt\% samples suggest the formation of reverse hexagonal phases $\left(H_{\|}\right) \cdot{ }^{36}$ The corresponding SAXS data (up to 50 $w t \%)$ reveal the growth of a sharp primary peak $\left(1 q^{*}\right)$, which is accompanied by the second $\left(2 q^{*}\right)$ and third order $\left(3 q^{*}\right)$ peaks at $50 \mathrm{wt} \%$ (Figure 2a). This is a clear indication of the $L_{\alpha}$ phase, with lattice-spacings $\left(d\right.$, calculated from $\left.1 q^{*}\right)$, which decrease from 11.4 to $7.1 \mathrm{~nm}$ for 40 to 50 wt\% (see Figure 2 and Figures S1-3 for fully indexed patterns of all AzoPS, and Table S4 for a summary of the calculated lattice parameters).
In comparison, $\mathrm{C}_{4} \mathrm{AzoOC}_{4} \mathrm{E}_{4}$, which has a slightly smaller headgroup, should have a larger packing parameter $(P \sim 0.39)$ and form lower curvature assemblies (closer to bilayers and lamellar sheets). POM confirms this prediction for 10-70 wt\% $\mathrm{C}_{4} \mathrm{AzoOC}_{4} \mathrm{E}_{4}$, with predominantly streaky textures observed, consistent with $L_{\alpha}$, see Figure $2 b .{ }^{35}$ The corresponding SAXS patterns show 2 peaks at $q$-spacing ratios of 1 and 2, with $d$ spacings that decrease from $13.1 \mathrm{~nm}(20 \mathrm{wt} \%)$, to $7.8 \mathrm{~nm}$ (30 $w t \%)$, to $6.5 \mathrm{~nm}$ (50 wt\%). This decrease in sheet-to-sheet distance is attributed to a reduction of water between adjacent layers as the surfactant concentration increases. ${ }^{37}$ At high concentrations (80-90 wt\%), densely packed, smoke-like textures are noted using POM, suggesting the presence of $L_{\alpha}$ or $H_{\|}$phases. ${ }^{35,38}$

$\mathrm{C}_{2} \mathrm{AzoOC}_{4} \mathrm{E}_{4}$ and $\mathrm{C}_{6} \mathrm{AzoOC}_{4} \mathrm{E}_{4}$ also appear to form almost exclusively $L_{\alpha}$ phases, with similar POM textures to $\mathrm{C}_{4} \mathrm{AzoOC}_{4} \mathrm{E}_{4}$ (Figures S1-2, ESI). Notably, $\mathrm{C}_{4} \mathrm{AzoOC}_{4} \mathrm{E}_{4}$ forms lamellar sheets even at $10 \mathrm{wt} \%$ (Figure 2b). Such hyperswollen lamellar phases have previously been reported for neutral surfactants when a critical alkyl chain $\left(C_{m}\right)$ /ethylene glycol $\left(E_{n}\right)$ ratio is reached $(2.4$ - 4). ${ }^{39}$ Within this critical range, the length of the surfactant's headgroup and tail are almost equal, which is thought to favor the formation of packed sheets with minimal curvature. ${ }^{39}$ The calculated $C_{m} / E_{n}$ ratios for all seven AzoPS are given in Figure 3. Interestingly, only $\mathrm{C}_{2} \mathrm{AzoOC}_{4} \mathrm{E}_{4}, \mathrm{C}_{4} \mathrm{AzoOC}_{4} \mathrm{E}_{4}$ and $\mathrm{C}_{6} \mathrm{AzoOC}_{4} \mathrm{E}_{4}$ give values that fall into the required range, suggesting that even this simple model is sufficient to predict the LLC phase behaviour of linear oxyethylene photosurfactants.

$\mathrm{C}_{4} \mathrm{AzoOC}_{4} \mathrm{E}_{2}$ and $\mathrm{C}_{8} \mathrm{AzoOC}_{8} \mathrm{E}_{4}$, which have the smallest headto-tail ratios and largest $P(\sim 0.52$ and $\sim 0.42$, respectively), are expected to form assemblies of flexible or planar bilayers. The POM textures and SAXS data for $\mathrm{C}_{4} \mathrm{AzoOC}_{4} \mathrm{E}_{2}$ up to $60 \mathrm{wt} \%$ are, however, difficult to interpret (Figure S3). At 10-20 wt\%, broad peaks in the SAXS data are observed at $q \approx 1.6 \mathrm{~nm}^{-1}$, indicating the presence of isotropic micellar phases $\left(I_{0}\right)$ with strong interparticle interactions. ${ }^{40}$ For $30-50$ wt\%, more defined first and second order peaks appear in the SAXS profiles, indicative of $L_{\alpha} \cdot{ }^{37}$ In contrast, at $30-60 \mathrm{wt} \%$ the POM images display smoke-like textures, consistent with the presence of the regular hexagonal phase $\left(H_{1}\right) .{ }^{38}$ Streaky $L_{\alpha}$ textures are noted for $70-80$ wt\% of $\mathrm{C}_{4} \mathrm{AzoOC}_{4} \mathrm{E}_{2}$. At 90 wt\% the smoke-like texture is recovered, which at such high concentrations can be assigned to the reverse hexagonal phase $H_{\|}$. In contrast, the significantly longer surfactant, $\mathrm{C}_{8} \mathrm{AzOOC}_{8} \mathrm{E}_{4}$, was relatively insoluble at the concentrations investigated and appeared crystalline by POM (Figure S4). This may be the result of reduced $\pi-\pi$ stacking between adjacent azobenzenes in the larger AzoPS, or its slightly reduced head/tail ratio. Finally, $\mathrm{C}_{8} \mathrm{AzoOC}_{4} \mathrm{E}_{4} 50$ wt\% was the only AzoPS to exhibit three sharp maxima in the SAXS pattern, at $q / q^{*}=1, \sqrt{ } 3$ and $\sqrt{ } 4$ at $50 \mathrm{wt} \%$, beginning at $q=0.82$ $\mathrm{nm}^{-1}$, accompanied by smoke-like textures in the POM images (Figure 2c), indicative of hexagonally-packed cylinders with modest translational order, $H_{1}$. The smaller peaks appearing from $q=0.56 \mathrm{~nm}^{-1}$ with $q / q^{*}=1,2$ also indicate a small contribution of $L_{\alpha}$.

Interestingly, $100 \% \mathrm{C}_{4} \mathrm{AzoOC}_{4} \mathrm{E}_{2}$ (Figure $\mathrm{S} 3$ ) and $\mathrm{C}_{4} \mathrm{AzoOC}_{4} \mathrm{E}_{6}$ (Figure $2 \mathrm{a}$ ), i.e. no solvent, are also birefringent, indicating the 


\begin{tabular}{|c|c|c|c|c|c|c|c|c|c|}
\hline $\mathrm{C}_{4} \mathrm{AzoOC}_{4} \mathrm{E}_{6}$ & & & & $L_{\alpha}$ & & $H_{\|}$ & & SmA & 2.3 \\
\hline $\mathrm{C}_{2} \mathrm{AzoOC}_{4} \mathrm{E}_{4}$ & $l_{0}$ & & & $L_{\alpha}$ & & $l_{0}$ & & - & 3 \\
\hline $\mathrm{C}_{4} \mathrm{AzoOC}_{4} \mathrm{E}_{4}$ & & & & $L_{a}$ & & & & - & .5 \\
\hline $\mathrm{C}_{6} \mathrm{AzoOC}_{4} \mathrm{E}_{4}$ & $l_{0}$ & & $H_{1}$ & & & $L_{a}$ & & - & 4 \\
\hline $\mathrm{C}_{8} \mathrm{AzoOC}_{4} \mathrm{E}_{4}$ & & $l_{0}$ & & $H_{1}$ & $r$ & $x$ & $x$ & - & 4.5 \\
\hline $\mathrm{C}_{8} \mathrm{AzoOC}_{8} \mathrm{E}_{4}{ }^{*}$ & $x$ & $x$ & $x$ & $x$ & $x$ & $x$ & $x$ & - & 5.5 \\
\hline $\mathrm{C}_{4} \mathrm{AzoOC}_{4} \mathrm{E}_{2}$ & & & & $H_{1}$ & & $L_{\mathrm{a}}$ & $H_{11}$ & SmA & 7 \\
\hline & & 20 & 30 & $40 \quad 50$ & 60 & $70 \quad 80$ & 90 & 100 & \\
\hline
\end{tabular}

Figure 3. The relationship between the AzoPS as a function of concentration and $\mathrm{C}_{m} / \mathrm{E}_{n}$ and liquid crystalline phase formation: isotropic (/0); lamellar $\left(L_{\alpha}\right)$; normal hexagonal $\left(H_{1}\right)$; reverse hexagonal $\left(H_{\Perp}\right)$ phases and thermotropic smectic $\mathrm{A}(\mathrm{SmA}) .{ }^{*}$ AzoPS did not dissolve in $\mathrm{H}_{2} \mathrm{O}$ at $\mathrm{T}=20^{\circ} \mathrm{C}$.

presence of thermotropic liquid crystals (TLCS). ${ }^{17}$ The POM images have pseudo-isotropic regions separated by oily streaks (Figure 2a), which is typically ascribed to a smectic phase (SmA) where the molecules are orientated normal to the plane. ${ }^{17}$ Tschierske et al. previously reported that a neutral AzoPS with 3 or 4 oxyethylene units within the headgroup $\left(\mathrm{C}_{12} \mathrm{OAzoE}_{n}(\mathrm{OH})_{2}\right.$, where $n=3$ or 4 ) formed TLC phases at high temperatures (100$170{ }^{\circ} \mathrm{C}$ ), but these were found to crystallise at lower temperatures. ${ }^{17}$ To the best of our knowledge $\mathrm{C}_{4} \mathrm{AzoOC}_{4} \mathrm{E}_{2}$ and $\mathrm{C}_{4} \mathrm{AzoOC}_{4} \mathrm{E}_{6}$ are the first examples of TLC phases for an AzoPS at room temperature.

Figure 3 summarises the relationship between molecular structure, concentration and head/tail ratio for AzoPS at $20^{\circ} \mathrm{C}$. Each phase has been assigned by careful consideration of the POM and SAXS data. We note that full interpretation of the SAXS data was challenging due to the fluid nature of LLC phases, which can result in swollen peaks that are difficult to isolate. However, it is evident that the length of both the head and tail groups has a significant effect on the presence and type of any LLC phases formed. As the number of oxyethylene units increases, the effective headgroup area $\left(a_{\mathrm{e}}\right)$ increases and, consequently, $P$ (and the $C_{m} / E_{n}$ ratio) decreases. Typically, as the concentration is increased there is a transition from a hexagonal to a lamellar phase, before re-assembly into a reverse hexagonal phase.

The temperature-dependence of AzoPS- $\mathrm{H}_{2} \mathrm{O}$ compositions identified as forming LLC phases was also investigated using POM. Each sample was slowly heated to $70{ }^{\circ} \mathrm{C}\left(1{ }^{\circ} \mathrm{C} \mathrm{min}-1\right)$ and then cooled to $20^{\circ} \mathrm{C}$ at the same rate. Typically, only minor changes to the LLC structure were observed upon heating. For a few AzoPS, a $H_{1}$ to $L_{\alpha}$ transition occurred, as shown for $\mathrm{C}_{4} \mathrm{AzoOC}_{4} \mathrm{E}_{6}$ (60 wt\%) between 15 and $20{ }^{\circ} \mathrm{C}$ in Figure 4. This order-order transition is likely to be related to the fact that the selectivity of water for the hydrophilic oxyethylene units over the hydrophobic domains decreases as the temperature is increased. ${ }^{16,41}$ A similar phase transition was also reported in the literature for the neutral AzoPS, $C_{4} A_{z o} C_{5} E_{7}$, (55 wt\% in water). ${ }^{16}$ For all AzoPS- $\mathrm{H}_{2} \mathrm{O}$, a temperature was reached in which any birefringence previously attributed to the formation of anisotropic LLC phase was lost, for example $C_{4} A_{20 O C} E_{4}(60$
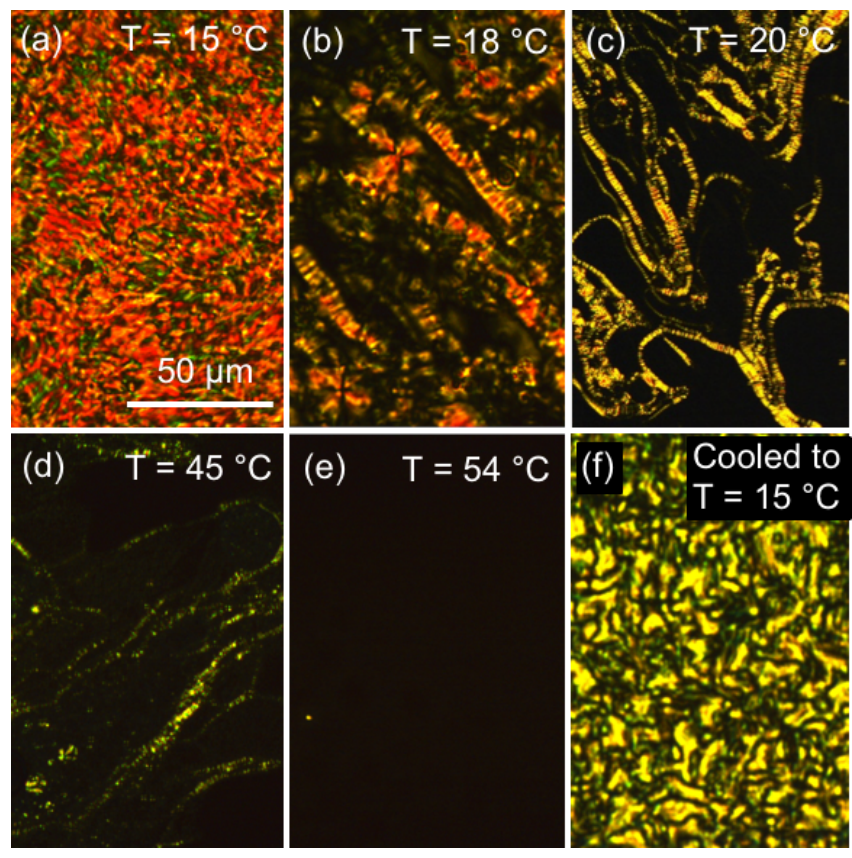

Figure 4. Temperature-dependant polarised optical micrographs of trans$\mathrm{C}_{4} \mathrm{AzoOC}_{4} \mathrm{E}_{6}(60 \mathrm{wt} \%)$ at (a) $15^{\circ} \mathrm{C}$, (b) $18^{\circ} \mathrm{C}$, (c) $20^{\circ} \mathrm{C}$, (d) $45^{\circ} \mathrm{C}$, (e) $54{ }^{\circ} \mathrm{C}$ and (f) cooled to $15^{\circ} \mathrm{C}$. Heating and cooling rates were $1{ }^{\circ} \mathrm{C} \mathrm{min}$. .

wt\%) lost all brightness at $45{ }^{\circ} \mathrm{C}$ (Figure 4). These melting temperatures are illustrated in the binary concentrationtemperature phase diagrams shown in Figure 5 and Figure $S 8$ in the ESI. In all cases, birefringence is recovered upon cooling. We note that on occasion the texture of the cooled sample was slightly dissimilar to the initial texture (e.g. Figure 4), which is attributed to the anchoring or organisation-inducing effect of the glass cover-slips. ${ }^{42}$ However, gentle agitation of the glass slides recovered the original textures. Finally, it is noted while $\mathrm{C}_{8} \mathrm{AzoOC}_{8} \mathrm{E}_{4}$ appeared to dissolve at $35^{\circ} \mathrm{C}$, it still showed no LLC phase formation by POM.

The effect of photoisomerisation on the LLC assemblies was also investigated. Trans-cis isomerisation upon irradiation with UV light $\left(\lambda_{\mathrm{ex}}=365 \mathrm{~nm}\right.$ ) was confirmed by UV/Vis absorption spectroscopy, with the observation of a characteristic change in the $\pi-\pi^{*}$ absorption band from $\sim 340$ to $\sim 440 \mathrm{~nm}$ upon conversion (see ESI, Figure S5). From these absorbance measurements it was possible to determine the extent of photoisomerisation, which ranged from $61-95 \%$ and $87-97 \%$ for the trans-to-cis and cis-to-trans isomerisation processes, respectively. It should be noted that these studies were performed on dilute systems $(<1 \mathrm{wt} \%)$ due to the negligible transmittance at the high concentrations used in this study. A full discussion of the photoisomerisation kinetics of dilute AzoPS solutions can also be found in the SI. Briefly, both photoisomerisation processes were found to obey first order kinetics, with the rate of isomerisation dependant on the length of oxyethylene glycol headgroup, with $\mathrm{C}_{4} \mathrm{AzoOC}_{4} \mathrm{E}_{6}$ having the fastest rate of isomerisation. This is a known effect of large electron-donating head-groups diminishing the energy barrier to isomerisation. ${ }^{25}$ 

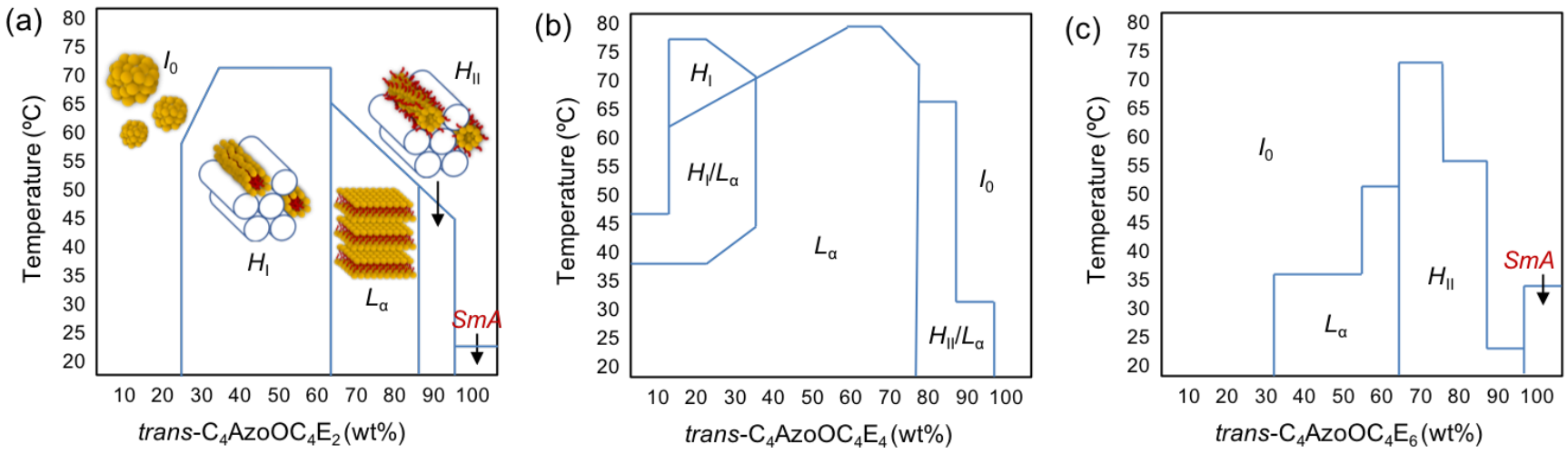

Figure 5. Temperature-concentration phase diagrams of the trans-isomers of a) $\left.C_{4} A_{z o O C} E_{2}, b\right) C_{4} A_{20 O C} E_{4}$ and c) $C_{4} A z o O C_{4} E_{6}$ as a function of concentration and temperature. $I_{0}, L_{\alpha}, H_{1}$ and $H_{\|}$represent the isotropic, lamellar and the normal and inverted hexagonal liquid crystalline phases, respectively, and SmA is the smectic $A$ thermotropic liquid crystalline phase.

For all AzoPS, global irradiation of the sample with UV light results in complete loss of brightness in the POM image, as shown for $\mathrm{C}_{2} \mathrm{AzOOC}_{4} \mathrm{E}_{4}$ and $\mathrm{C}_{8} \mathrm{AzoOC}_{8} \mathrm{E}_{4}$ in Figure 6. This indicates that the cis-isomers do not form anisotropic LLC phases. A similar order-disorder transition was noted for the neutral AzoPS, $\mathrm{C}_{4} \mathrm{AzOC}_{5} \mathrm{E}_{7}$, which formed an ordered $H_{1}$ phase at $50 \mathrm{wt} \%$ surfactant before UV-irradiation, ${ }^{16}$ and transformed into an isotropic state upon photoirradiation. The highlyordered phases formed by tightly-packed trans-isomers are likely to be disrupted by a change in molecular geometry (rodlike to "bent") upon photoisomerisation. This effect may also be a consequence of the difference in hydrophobicity of the two isomers, with the trans-isomer being considerably more hydrophobic than the cis-form (the dipole moments are $\sim 0 \mathrm{D}$ and $\sim 3 D$, respectively ${ }^{43}, 44$ ). It is therefore unsurprising that $\mathrm{C}_{8} \mathrm{AzoOC}_{8} \mathrm{E}_{4}$, which has the longest tail, appears crystalline in the trans-state at high concentration, but dissolves upon photoirradiation to the cis-dominant isomer (Figure 6a). In contrast, $\mathrm{C}_{2} \mathrm{AzoOC}_{4} \mathrm{E}_{4}$, with the shortest chain, becomes less soluble and undergoes phase separation in the cis-form at the same concentration (Figure 6b). One explanation for this unexpected behaviour could be the formation of inverse micelles, in which the alkyl tails now represent the head group, thus rendering the cis-isomer immiscible with water. Unfortunately, it was not possible to perform SAXS measurements on the cis-isomers due to their inherent instability under the intense X-ray beam, resulting in reverse isomerisation of the AzoPS to its trans-native state (Figure S7). Future SAXS measurements of the cis-isomers could be performed on a low-power benchtop instrument in order to minimise these effects. Nevertheless, it should be noted that the phases were all recovered upon reverse switching to the trans-form with blue light $\left(\lambda_{\mathrm{ex}}=465 \mathrm{~nm}\right)$. Finally, the POM images in Figure 6 also highlight the ability to mask between irradiated and non-irradiated sample (dashed line), leading to local deformation of the LLC structure only at the exposed areas.

\section{Conclusions}

\section{(a) $\mathrm{C}_{8} \mathrm{AzoOC}_{8} \mathrm{E}_{4}(50 \mathrm{wt} \%)$}

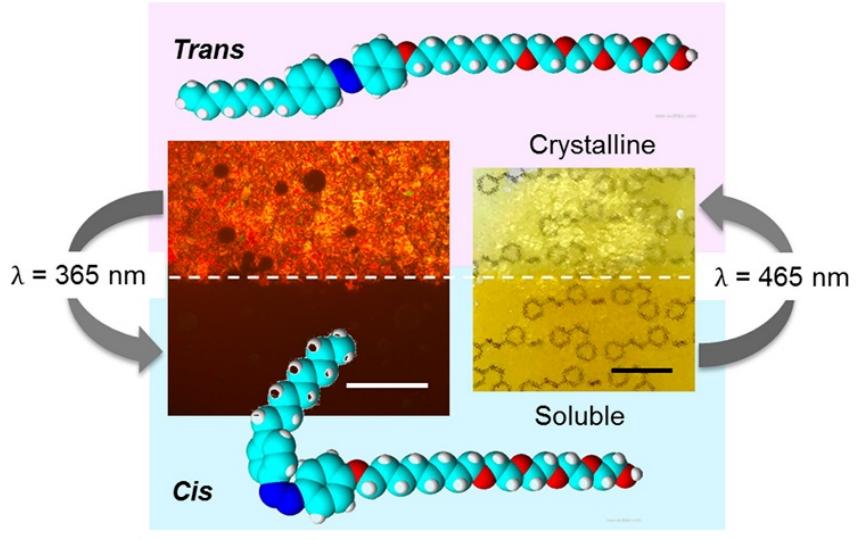

(b) $\mathrm{C}_{2} \mathrm{AzoOC}_{4} \mathrm{E}_{4}$ (50 wt\%)

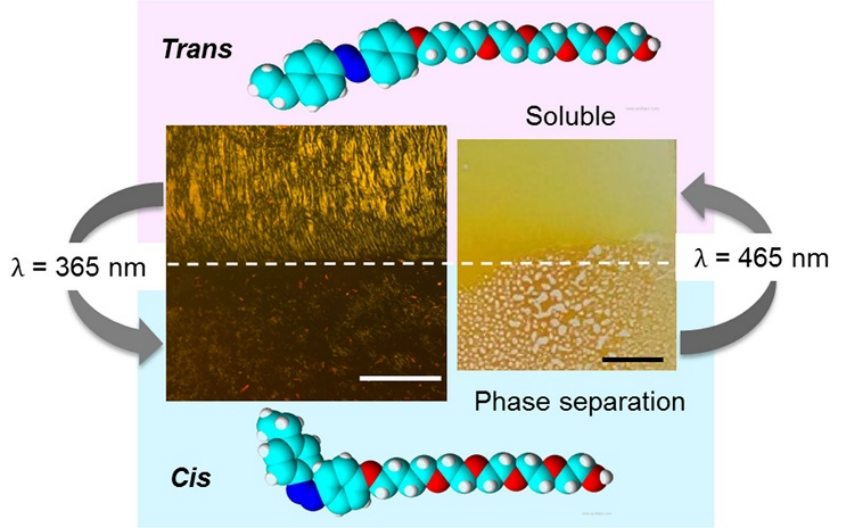

Figure 6. Polarised optical micrographs (left) and photographs (right) of (a) $\mathrm{C}_{8} \mathrm{AzOOC}_{8} \mathrm{E}_{4} \quad(50 \mathrm{wt} \%)$ and (b) $\mathrm{C}_{2} \mathrm{AzOOC}_{4} \mathrm{E}_{4}$ (50 wt\%) in $\mathrm{H}_{2} \mathrm{O}$ after photoisomerisation. Scale bars represent $50 \mu \mathrm{m}$ and $5 \mathrm{~mm}$ for the POM and the photographs, respectively. The dashed, white lines represent the physical mask used to control the irradiation area. The azobenzene structures in background of the photo (a) serve only to emphasise the difference in transparency between irradiated areas. The molecular models show the estimated 3D structures of the AzoPS (see the Experimental Section for more details): oxygen (red), hydrogen (white), nitrogen (blue), carbon (teal).

In summary, a series of neutral azobenzene photosurfactants with different alkyl tails and polar head-groups have been 
investigated to assess the structure-self-assembly relationships at concentrations significantly above the critical micelle concentration. The simple packing parameter was used as a starting point to design AzoPS structures that were expected to assemble into LLC mesophases in this concentration regime. However, we observed that in the trans-form the type of LLC phase that is formed is primarily controlled by the ratio of hydrophilic head/hydrophobic tail length, rather than the packing parameter. By careful selection of the alkyl-chain length and the number of ethylene oxide units in the head-group, the dimensions of the LLC phases could be tuned through this relatively simple approach. We also observed that the packing parameter could not adequately account for the large change in the dimensions of the surfactant upon photoisomerisation to the cis-form. Irradiation with UV light was shown to quickly disperse any anisotropic LLC phases formed, which is attributed to the imperfect packing of the cis-isomers. This process could be reversed with blue light to recover the original LLC phase. Moreover, using a simple mask, the LLC phase could be selectively deformed with spatial control, demonstrating the potential of light as an elegant, non-invasive trigger for functional LLC phases. Notably, we have demonstrated that neutral AzoPS exhibit a rich liquid crystalline phase behaviour, which ranges from the common hexagonal and lamellar phases, to thermotropic liquid crystals at room temperature. Furthermore, these single-component LLC phases endowed with photoresponsive properties pave the way for new bio- and environmentally-friendly functional materials to be developed, for example as photoresponsive templates ${ }^{45}$ or drug delivery systems. ${ }^{46}$

\section{Conflicts of interest}

There are no conflicts to declare.

\section{Acknowledgements}

We acknowledge Diamond Light Source for time on Beamline B21 under Proposal SM16235. We thank Lisa Fruhner and Camille Blayo for their help with SAXS measurements on GALAXI and B21, respectively, and $\operatorname{Dr}$ Marie-Sousai Appavou for help with POM. Dr Martin Feeney and Dr John O'Brien are thanked for their help with MS and NMR measurements. EAK thanks the Irish Research Council for a Government of Ireland postgraduate scholarship. This work was supported in part by an Isaac Newton Trust/University of Cambridge Early Career Support Scheme grant. Support from COST action MP1202 is gratefully acknowledged.

\section{Notes and references}

1 R. Negrini, W.-K. Fong, B. J. Boyd and R. Mezzenga, Chem. Commun., 2015, 51, 6671-6674.

2 X. Mulet, B. J. Boyd and C. J. Drummond, J. Colloid Interface Sci., 2013, 393, 1-20.

3 E. Tunkara, C. Albayrak, E. O. Polat, C. Kocabas and Ö. Dag, ACS Nano, 2014, 8, 11007-11012.
4 S. Besson, T. Gacoin, C. Ricolleau, C. Jacquiod and J.-P. Boilot, J. Mater. Chem. C, 2003, 13, 404-409.

5 C. G. Goltner and M. Antonietti, Adv. Mater., 1997, 9, 431436.

6 J. Jennings, B. Green, T. J. Mann, C. A. Guymon and M. K. Mahanthappa, Chem. Mater., 2018, 30, 185-196.

7 S. Hara, H. Wada, A. Shimojima and K. Kuroda, ACS Nano, 2017, 11, 5160-5166.

8 S. Akbar, J. M. Elliott, M. Rittman and A. M. Squires, Adv. Mater., 2013, 25, 1160-1164.

9 Y. Huang and S. Gui, RSC Advances, 2018, 8, 6978-6987.

10 A. Klaiber and S. Polarz, ACS Nano, 2016, 10, 10041-10048.

11 M. A. C. Stuart, W. T. S. Huck, J. Genzer, M. Müller, C. Ober, M. Stamm, G. B. Sukhorukov, I. Szleifer, V. V. Tsukruk, M. Urban, F. Winnik, S. Zauscher, I. Luzinov and S. Minko, Nat. Mater., 2010, 9, 101-113.

12 J. J. Vallooran, S. Bolisetty and R. Mezzenga, Adv. Mater., 2011, 23, 3932-3937.

13 W.-K. Fong, T. Hanley and B. J. Boyd, J. Control. Release, 2009, 135, 218-226.

14 14. A. Yaghmur, L. Paasonen, M. Yliperttula, A. Urtti and M. Rappolt, J. Phys. Chem. Lett., 2010, 1, 962-966.

15 K. J. Tangso, W.-K. Fong, T. Darwish, N. Kirby, B. J. Boyd and T. L. Hanley, J. Phys. Chem. B, 2013, 117, 10203-10210.

16 S. Peng, Q. Guo, P. G. Hartley and T. C. Hughes, J. Mater. Chem. C, 2014, 2, 8303-8312.

17 X. Tan, R. Zhang, C. Guo, X. Cheng, H. Gao, F. Liu, J. R. Bruckner, F. Giesselmann, M. Prehm and C. Tschierske, J. Mater. Chem. C, 2015, 3, 11202-11211.

18 R. Yang, S. Peng, W. Wan and T. C. Hughes, J. Mater. Chem. C, 2014, 2, 9122-9131.

19 S. Peng, Q. Guo, T. C. Hughes and P. G. Hartley, Langmuir, 2014, 30, 866-872.

20 Z. Wu, R. Xue, M. Xie, X. Wang, Z. Liu, M. Drechsler, J. Huang and Y. Yan, J. Phys. Chem. Lett., 2018, 9, 163-169.

21 E. Chevallier, C. Monteux, F. Lequeux and C. Tribet, Langmuir, 2012, 28, 2308-2312.

22 J. Calbo, C. E. Weston, A. J. P. White, H. S. Rzepa, J. ContrerasGarcía and M. J. Fuchter, J. Am. Chem. Soc., 2017, 139, 12611274.

23 J. Choi, W. Cho, Y. S. Jung, H. S. Kang and H.-T. Kim, ACS Nano, 2017, 11, 1320-1327.

24 S. Jaekel, A. Richter, R. Lindner, R. Bechstein, C. Nacci, S. Hecht, A. Kühnle and L. Grill, ACS Nano, 2018, 12, 1821-1828.

25 H. M. D. Bandara and S. C. Burdette, Chem. Soc. Rev., 2012, 41, 1809-1825.

26 E. A. Kelly, J. E. Houston and R. C. Evans, Soft Matter, 2019, 15, 1253-1259.

27 R. Lund, G. Brun, E. Chevallier, T. Narayanan and C. Tribet, Langmuir, 2016, 32, 2539-2548.

28 J. N. Israelachvili, D. J. Mitchell and B. W. Ninham, J. Chem. Soc., Faraday Trans. 2, 1976, 72, 1525-1568.

29 R. Nagarajan, Langmuir, 2002, 18, 31-38.

30 C. Blayo, J. E. Houston, S. M. King and R. C. Evans, Langmuir, 2018, 34, 10123-10134.

31 R. F. Tabor, R. J. Oakley, J. Eastoe, C. F. J. Faul, I. Grillo and R. K. Heenan, Soft Matter, 2009, 5, 78-80.

32 T. Shang, K. A. Smith and T. A. Hatton, Langmuir, 2003, 19, 10764-10773.

33 E. Kentzinger, M. Krutyeva and U. Rücker, J. large-scale Res. Facil., 2016, 2, A61.

34 ACD/ChemSketch, version 2017.2.1, Advanced Chemistry Development, Inc., Toronto, ON, Canada, www. acdlabs, 2017.

35 S. T. Hyde, Handbook of Applied Surface and Colloid Chemistry John Wiley \& Sons, Ltd., 2001.

36 J. Borné, T. Nylander and A. Khan, Langmuir, 2000, 16, 1004410054. 
37 J.-C. P. Gabriel, F. Camerel, B. J. Lemaire, H. Desvaux, P. Davidson and P. Batail, Nature, 2001, 413, 504-508.

38 Y. Zhao, X. Yue, X. Wang and X. Chen, J. Colloid Interface Sci., 2013, 389, 199-205.

39 Y. Uchida, T. Nishizawa, T. Omiya, Y. Hirota and N. Nishiyama, J. Am. Chem. Soc., 2016, 138, 1103-1105.

40 L. Wang, R. Tabor, J. Eastoe, X. Li, R. K. Heenan and J. Dong, Phys. Chem. Chem. Phys., 2009, 11, 9772-9778.

41 T. P. Lodge, B. Pudil and K. J. Hanley, Macromolecules, 2002, 35, 4707-4717.

42 T. Ichikawa, T. Kato and H. Ohno, J. Am. Chem. Soc., 2012, 134, 11354-11357.

43 K. G. Yager and C. J. Barrett, J. Photochem. Photobiol. A, 2006, 182, 250-261.

44 G. S. Hartley and R. J. W. Le Fevre, J. Chem. Soc., 1939, 531535.

45 J. Wei, Y. Liu, J. Chen, Y. Li, Q. Yue, G. Pan, Y. Yu, Y. Deng and D. Zhao, Adv. Mater., 2014, 26, 1782-1787.

46 W.-K. Fong, T. L. Hanley, B. Thierry, N. Kirby, L. J. Waddington and B. J. Boyd, Langmuir, 2012, 28, 14450-14460. 